\title{
e-Phaïstos
}

e-Phaïstos

Revue d'histoire des techniques / Journal of the history

of technology

IV-2 | 2015

Patrimoines de l'eau

\section{La semi-insularité de Safi. Les défis de la patrimonialisation}

Semi insularity of Safi. Challenges of patrimonialization

\section{Ana Sofia Neno Leite}

\section{OpenEdition}

Journals

Édition électronique

URL : http://journals.openedition.org/ephaistos/777

DOI : 10.4000/ephaistos. 777

ISSN : 2552-0741

Éditeur

IHMC - Institut d'histoire moderne et contemporaine (UMR 8066)

Édition imprimée

Date de publication : 1 octobre 2015

ISSN : 2262-7340

Référence électronique

Ana Sofia Neno Leite, "La semi-insularité de Safi. Les défis de la patrimonialisation », e-Phaïstos [En ligne], IV-2 | 2015, mis en ligne le 01 octobre 2015, consulté le 19 avril 2019. URL : http:// journals.openedition.org/ephaistos/777 ; DOI : 10.4000/ephaistos.777

Ce document a été généré automatiquement le 19 avril 2019

Tous droits réservés 


\title{
La semi-insularité de Safi. Les défis de la patrimonialisation
}

Semi insularity of Safi. Challenges of patrimonialization

\author{
Ana Sofia Neno Leite
}

\section{La semi-insularité de Safi, une condition politique et historique}

1 La ville de Safi est mentionnée, depuis le $\mathrm{XI}^{\mathrm{e}}$ siècle, comme une ville très peuplée et un port actif. Sous le pouvoir Almohade, pendant le XII siècle, la ville a été dotée d'une muraille et d'une casbah, ainsi que d'une grande mosquée et d'un ribat religieux (résidence du Morabitin). La structure urbaine de Safi a été enrichie, pendant la période Mérinide, avec la construction d'une medrassa (l'école coranique) et d'un bimaristan (l'hôpital). Safi était déjà à l'époque du roi portugais D. Afonso V (1438-1481) une ville riche, avec un des ports les plus actifs du Maroc. De l'installation d'une factorerie en Arguin (actuelle Mauritanie) a surgi le besoin de créer des biens d'échanges pour l'or subsaharien, Safi était alors un choix potentiel pour l'installation d'un entrepôt commercial.

2 À la fin du $\mathrm{XV}^{\mathrm{e}}$ siècle, Safi n'était ni sous le pouvoir du Royaume de Fès, ni de celui des émirs de Marrakech. La ville se faisait attaquer souvent par la noblesse andalouse et comme elle n'avait pas l'aide interne du Royaume, elle demanda la protection de D. João II en 1488, en acceptant la suzeraineté et le protectorat du Portugal.

Une série d'éléments architecturaux et urbains ont été intégrés à Safi, pendant le Protectorat portugais, et surtout au moment de la domination effective de la couronne portugaise (1508-1541). Quelques éléments sont encore présents dans la ville et sont passés par un processus de patrimonialisation pendant la période moderne.

De nos jours, Safi continue à être conservée dans une espèce de semi-insularité par rapport au territoire national. Ce phénomène est en partie un effet de sa situation géographique qui favorise les flux humains vers Marrakech, Essaouira ou Casablanca, en 
laissant la ville de Safi hors des circuits touristiques et commerciaux principaux. Le port continue néanmoins à avoir un rôle fondamental dans la structure et l'organisation de la ville.

Dans la correspondance à l'époque du protectorat entre acteurs locaux et agents de l'administration coloniale de la Résidence Générale, on trouve des mentions renvoyant à une espèce d'aliénation et d'abandon de la ville. À propos d'une visite du Ministre Plénipotentiaire de l'administration coloniale en 1948, M. Girard, délégué au $3^{\mathrm{e}}$ Collège, a proféré une petite allocution, en représentant la population de Safi et en demandant l'intervention du gouvernement pour le développement de la ville : «Safi a trop souvent été oubliée et nous aimerions, Monsieur le Ministre, que votre passage marque justement le début d'une période au cours de laquelle les aménagements indispensables que la collectivité désire recevront un début d'exécution $»^{1}$.

6 Selon un autre rapport, le développement du port, entraîné par la création de l'O.C.P., la compagnie des phosphates, à partir des années 1940, a provoqué une rupture dans l'équilibre de la ville « une faille, une rupture très nette entre le bled et la ville $»^{2}$.

7 Dans les années cinquante, la ville de Safi était considérée comme le deuxième port marocain et la deuxième ville industrielle du Maroc, dans les rapports officiels. Sa superficie de $40 \mathrm{~km}^{2}$ était comparée à celle de Lyon. Les rapports montrent aussi une énorme croissance de la population, une duplication par rapport aux chiffres du recensement du début des années de 1930, et la prévision d'une croissance continuelle. Mais, si un fort développement des secteurs de la pêche et de l'industrie s'annonçait, une instabilité sociale était une menace pour le pouvoir central :

- Cette population - composée, à côté d'une minorité de citadins anciens, d'un ramassis de gens des tribus des Régions de Marrakech et d'Agadir, - est pauvre, mouvante, vouée au chômage (I) permanent et saisonnier ;

- Politiquement, SAFI est réputé être le 2ème Centre de l'ISTIQLAL. Le « lien de la pêche et de la conserve» facilite la diffusion des propagandes et des mots d'ordre, explique la soudaineté des réactions ;

- Rappelons les événements de :

- 1951 (le personnel du Territoire est assiégé à "la Kechla" pendant 48 heures),

- 1952 (la Mahakma ${ }^{3}$ est assaillie ; le Pacha doit faire le coup de feu),

- Août 1954,

- 36 attentats terroristes causant 4 morts et 10 blessés ont été commis depuis Décembre 1953. $[\ldots]$

8 (I) Pour lutter contre le chômage, la création d'un Bureau de Placement a été demandée avec la participation de la Municipalité et de la Division du Travail.

9 D'autre part, un plan d'emploi de la main d'œuvre a été établi ${ }^{4}$.

10 Le rapport entre la ville de Safi et le pouvoir central a été très fragile à plusieurs moments de son histoire. Même après l'indépendance, cette fragile relation avec le territoire national reste une caractéristique de la ville. Néanmoins, on peut toujours reconnaitre à Safi une forte relation avec la mer et les nombreuses activités qui l'ont utilisé comme ressource première pour le développement local. À partir des années 1960, l'implantation d'un complexe chimique traitant le phosphate de Youssoufia a imprimé une nouvelle dynamique au port de Safi, une activité qui reste toujours de grande importance. Les industries chimiques ont créé une considérable source d'emploi pour Safi et en même temps, elles ont contribué à la consolidation du port. L'impact environnemental a été très 
néfaste pour l'image de la ville à Safi : « Le complexe de Safi est malheureusement terriblement polluant, ce qui ne contribue pas à améliorer l'image d'une ville en difficulté $»^{5}$.

11 Selon Jean-François Troin, la petite région de Safi « est à la fois fortement dépendante d'attractions septentrionales et en même temps beaucoup plus tournée vers la mer que ses voisines immédiates (Doukkala et Chiadma) $»^{6}$. L'économie de la région des Abda repose largement sur l'exploitation de l'Océan Atlantique, la pêche, le traitement du poisson et des activités portuaires; mais une crise du secteur de la pêche ces dernières années a eu des effets importants (et néfastes) dans l'équilibre économique et social de la ville.

Du fait de ces spécificités, le patrimoine de Safi est dans une situation particulièrement sensible et doit être compris en tenant compte des conditions de ce contexte géographique, urbain et social. La condition maritime de Safi a été depuis son origine une source de possibilités, mais aussi également une source de contraintes pour son développement et pour les pratiques spatiales quotidiennes de ses populations dans la ville, ce qui se ressent dans les discours qu'on analyse ici, à plusieurs niveaux.

Comme on peut constater à partir de ces textes, l'isolement et le sentiment de «semiinsularité » n'est pas une condition moderne, mais a toujours été présente à travers l'histoire de Safi. Je cite un passage de Troin à ce propos :

«Safi constitue l'unique grande ville régionale (262 276 hab.) mais alors qu'elle pourrait régner sur un territoire qui l'enserre parfaitement, elle est quelque peu coupée de son espace régional. Ceci peut en partie s'expliquer par les cycles d'expansions et de replis qu'elle a historiquement subis et qui ne lui ont pas permis d'affermir son contrôle spatial. Port actif au Moyen-Âge, elle est occupée par les Portugais au $\mathrm{XVI}^{e}$ siècle, et son rôle de forteresse étrangère fait fuir les habitants et coupe la ville une première fois de son arrière-pays rural. Ville saadienne, devenue port de Marrakech au XIXe et début XXe siècle, elle va voir en suite son commerce décliner. Sous le protectorat français, elle est à nouveau disjointe de son hinterland (Hajouj, 1989), les phosphates s'exportant par Casablanca et le développement de la pêche et des conserveries l'ouvrant sur la mer bien plus que vers la terre ${ }^{7}$."

Les relations commerciales et le drainage des produits agricoles de l'arrière-pays se sont tournés vers d'autres pôles, comme Essaouira, Casablanca, ou même Marrakech. La ville de Safi et son arrière-pays restent toujours dos tournés. Cela est mis en évidence lorsqu'on comprend que le réseau routier privilégie une liaison directe entre Essaouira et Casablanca qui exclut Safi de ce parcours.

Le développement urbain de Safi a donc toujours été très dépendant de la relation établie entre ce territoire et la mer, d'après ce qu'on vient d'analyser. Le patrimoine de la ville, inscrit dans cette logique, contribue à la création d'une narration spécifique, constituée par plusieurs regards et par des discours polyphoniques ${ }^{8}$. Certains éléments architecturaux servent à construire "la fiction que crée les lecteurs, qui rend la complexité de la ville lisible et qui immobilise sa mobilité opaque dans un texte transparent $"$. À travers ces éléments architecturaux, qu'on peut trouver dans les discours sur l'espace et le patrimoine, depuis le début du Protectorat, on peut faire une lecture, ou plutôt plusieurs lectures complémentaires, sur la ville de Safi. 


\section{L'imaginaire du patrimoine safiot, identification et classement}

«Il est plusieurs manières de chercher à lire l'histoire du patrimoine. Le fait patrimonial, défini largement, touche à la destinée générale des œuvres et des objets matériels, à la représentation culturelle d'une collectivité, à l'interprétation du passé. Ainsi son histoire participe-t-elle de l'histoire matérielle des objets, de celle des symboles identitaires, des réflexions sur l'authenticité ${ }^{10}$. »

En 1952, la Chambre Mixte Française d'Agriculture, de commerce et d'industrie de Safi a édité un petit bulletin sur Safi pour promouvoir l'image d'une ville prospère avec de fortes possibilités de développement économique. En plus des petits articles sur l'industrie, l'agriculture et le commerce, apparaissent aussi des articles sur les sports, les arts et l'histoire de Safi. Un de ces articles s'intitule "Monuments et points de vue ». L'association d'idées dans le titre choisi est déjà un signe intéressant du rapport entre le patrimoine d'une ville et son iconographie, ce qui constitue déjà une narration. La fiction de la ville est ainsi lisible à travers les choix de ce discours, à travers un passé monumental qui domine la ville.

Les objets patrimoniaux énumérés comme des icônes de Safi ont été : le Château de Mer, la Chapelle Portugaise, la Kechla, la Colline des Potiers, le Marabout de Sidi-Bouzid. Alors que les monuments servent de base à un discours sur les spécificités identitaires de cette ville, le discours se tourne aussi vers l'idée d'observation en vol d'oiseau, une espèce de points phare qui dominent visuellement et symboliquement la ville, vers la mer ou à partir de la mer :

«- Le Château de Mer. - Spécimen unique de l'art emmanuélin au Maroc, le Château de Mer est le plus important vestige de l'occupation Portugaise. [...]

Des terrasses, le regard s'étend sur le panorama d'ensemble de Safi.

- La Chapelle Portugaise. - Située en Médina, derrière la Cour de Habous, cette chapelle dédiée à Sainte Catherine, est le seul monument inspiré de l'Art Gothique en Afrique du Nord.

- La Kechla. - Ancienne citadelle, des sultans, construite au 18ème siècle, la Kechla est, sans conteste, le plus beau monument de Safi. [...]

Du premier étage où sont maintenant installés les Bureaux du Territoire on jouit d'un admirable panorama sur la ville et la mer.

- La Colline des Potiers. - La Colline de Potiers, elle aussi offre un remarquable point de vue sur la Kechla, le souk et la mer. [...]

- Le Marabout de Sidi-Bouzid. - Situé au sommet de la grande Falaise qui surplombe la mer de cent mètres, le Marabout de Sidi Bouzid est entouré de jardins. C'est un des plus vastes et plus pittoresques points de vue de la côte ${ }^{11}$. »

Dans cette relation étroite de la ville à la mer qui est aussi en dialogue avec le patrimoine, l'architecture et l'urbanisme, un des éléments qu'on va trouver plus transversalement dans l'analyse des archives de la période de l'occupation française, aussi bien que dans les discours actuels, est effectivement le Château de Mer. Cet élément est une des grands icônes du patrimoine de Safi. Sa relation avec la mer l'expose à une condition iconographique pour la ville, mais aussi à un péril quasi permanent face aux agressions naturelles. L'écroulement récent d'une de ses tours, par les effets de l'érosion provoqués par des vagues maritimes et par le manque d'une politique active de gestion patrimoniale et de sauvegarde, a remis à l'ordre du jour la discussion de l'importance du patrimoine dans la ville. Mais on trouve presque les mêmes préoccupations depuis les premières 
démarches de patrimonialisation et dans les discours plus contemporains sur le patrimoine de Safi.

Les premières politiques de patrimonialisation à Safi, dans les premiers années du Protectorat Français, ont proposé le classement des éléments architecturaux considérés les plus importants pour l'identité urbaine safiote. Une quête pour l'identification des monuments historiques à préserver a été mise en place par l'administration coloniale. En 1921, l'Inspecteur Régional de la Région Sud envoyait un document au Chef du Service des Beaux-Arts et Monuments Historiques, où il décrivait l'intérêt particulier de quelques éléments architecturaux sur lesquels il soumettait aussi des projets de classement :

« $1^{\circ}$ Les remparts :

Les remparts comprennent, outre l'enceinte de défense entourant la Médina avec ses murailles, tours et portes, le monument dit de la Kechla actuellement occupé par le Contrôle Civil, et le vieux château-fort situé sur la mer à côté du port. Tout ce système de fortification est nettement portugais ; Il n'y a pas, semble-t-il, grand chose à faire pour le moment dans toute la partie entourant la ville, mais c'est dans le vieux château-fort de la mer que nous aurons à travailler. [...]

[...] c'est dans les tours, dans les salles voûtées intérieures, et dans les magasins à munitions, aux créneaux, qu'il est nécessaire et urgent de faire des travaux d'entretien et de réfection [...] Il y aurait d'ailleurs quelque chose tout à fait charmant à faire avec ce vieux château-fort en s'inspirant peut-être de ce qui a été fait à la Médersa à Rabat. L'intérieur en est actuellement occupé par des maisons indigènes malpropres et délabrées, par des latrines qui empestent le quartier et enfin, chose assez imprévue, par un dispensaire médical. On pourrait dès maintenant envisager la disparition de toutes ces verrues et préparer l'aménagement à cet endroit d'un jardin et d'un musée.

$2^{\text {o }}$ La vieille mosquée située sur le terre-plein des Contrôles Civil en face de la Kechla;

Cette vieille mosquée complète très heureusement l'admirable paysage que l'on a, à ses pieds, du haut des balcons de la Kechla. Elle a été malheureusement décrépite, il y a peu de temps, par le Service des Habous, ce qui lui ôte momentanément son caractère de vétusté et de délabrement qui était son plus grand charme, et c'est pour éviter que de pareilles erreurs se renouvellent, que je vous propose son classement. [...]

3ํㅡㄹéglise portugaise :

Nous arrivons maintenant à la question capitale.

[...] Il ne s'agit plus ici d'architecture militaire comme dans les divers systèmes de remparts ou même comme dans la grande salle d'armes de Mazagan ; il ne s'agit pas non plus d'un simple souvenir historique comme à l'Église de l'Assomption, mais d'un petit chef-d'œuvre d'art manuélin égaré en Afrique, miraculeusement échappé aux déprédations des indigènes et qui devrait déjà être non seulement classé mais même dégagé, remis en état, ouvert librement au public. Cet édifice est actuellement occupé par un bain maure, [...].

[..] D'abord, les deux salles actuellement visibles sont-elles les seules existantes et n'y-a-t-il pas d'autres, noyées à l'intérieur des maisons voisines ? S'il n'y a que deux salles, les travaux consisteraient d'abord dans la démolition des cloisons établies pour les besoins du hammam, dans la réfection de la voûte effondrée, au-dessus de la chaufferie, et ensuite, dans les travaux de consolidation de la voûte de la salle principale; celle-ci doit être, extérieurement, en mauvais état, puisque les indigènes ont jugé bon de la soutenir par un gros pilier en briques qu'il faudrait, bien entendu, supprimer, parce qu'il encombre la salle et masque en partie les grandes armoiries sculptées dans les pierres d'appareil et qui constituent le plus bel ornement de l'édifice ${ }^{12}$.» 
Dans le cas de la province de Safi, à peine quatorze monuments ont été classés comme patrimoine national jusqu'à nos jours. Tous ces classements remontent à la période du Protectorat Français. Le premier classement effectué à Safi a été le Quartier des Potiers en 1920 (dahir du 19 novembre 1920 portant classement, B.O. n 423 du 23 Novembre 1920 P. 16.) et le dernier a été la résidence du Caïd des tribus Abda, le Dar-SI-Aîssa en 1954 (Arrêté Viziriel du 2 décembre 1953, portant classement, B.O. n 2150 du 8 janvier 1954 - P.41).

Pour l'administration coloniale française, les objets patrimoniaux identifiés et les objets de classement et de sauvegarde n'ont été que des monuments d'origine locale, vernaculaire, mais aussi tout le patrimoine attribué à la période d'occupation portugaise. En ce qui concerne ce cas d'étude les monuments et sites classés depuis le début des démarches de patrimonialisation jusqu'à nos jours sont les suivants :

- Château de mer portugais (dahir 07/11/1922 portant classement, B.O nº du 21/11/1922- P. 1642) ;

- Kechla de Safi (dahir du 25 novembre 1922 portant classement, B.O. nº 528 du 5 décembre 1922 P. 1718.) ;

- Remparts de Safi (dahir du 3 juillet 1923 portant classement, B.O. n 560 du 17 juillet 1923 . P. 871) ;

- Église portugaise de Safi (dahir du 21 janvier 1924 portant classement, B.O. n 593 du 26 février 1924 P.382) ;

- Zones de servitude de protection artistique autour du château de mer portugais de Safi (dahir du 20 février 1924, B.O. n 596 du 25 mars 1924 P. 544);

- Ruines de l'église portugaise de l'impasse Sidi Abdelkrim à Safi (dahir du 7 mai 1930 portant classement B.O. $n^{\circ} 921$ du 2 juin 1930- P.735).

À l'exception de la Colline des Potiers et des monuments et sites d'origine portugaise, toutes les autres classifications ne se trouvent pas dans les limites urbaines de Safi. Cela veut dire que les objets patrimoniaux qui représentent la ville de Safi sont surtout cet imposant patrimoine d'origine étrangère, qui a été identifié et patrimonialisé par le colon et qui reste comme une icône de la ville jusqu'à nos jours.

La vieille mosquée mentionnée dans la proposition de classement citée précédemment n'a jamais été classée, au même titre que d'autres éléments islamiques d'une importance très révélatrice du point de vue historique et artistique, et aussi dans l'imaginaire safiot. Le minaret qui reste de la grande mosquée Almohade, et qui a été détruit pendant les années de l'occupation portugaise pour l'implantation de la cathédrale, assume un caractère de référence très pertinent dans l'ancienne médina de Safi. Ce minaret est un exemple remarquable de l'architecture Almohade et pourrait avoir servi de champs d'expérimentation pour la conception des minarets de la Tour Hassan à Rabat et de la Koutoubia à Marrakech, des importants symboles nationaux et supra-nationaux du Maroc, selon la thèse de l'archéologue safiot et actuel délégué provincial de la direction du patrimoine cultural, Said Chemsi.

Également très intéressant, est le fait que les discours actuels des gens de Safi sur les problèmes identifiés et les valeurs accordés, demeurent de façon très similaire à ceux du début du $\mathrm{XX}^{\mathrm{e}}$ siècle. L'isolement de la ville par rapport à l'ensemble du territoire national a peut-être imprimé aussi une stagnation dans les pratiques patrimoniales, ainsi qu'une adoption et adaptation de certains discours.

L'héritage du discours colonial, peut, de mon point de vue, avoir influencé les pratiques spatiales et les représentations du patrimoine culturel de Safi. C'est encore dans les 
couches les plus intellectualisées et les plus européanisées qu'on trouve les discours les plus visibles et les plus médiatisés. Ces éléments architecturaux qui constituent le patrimoine classé de Safi, majoritairement des éléments coloniaux portugais, constituent une espèce de scène présente dans les pratiques quotidiennes, mais où l'interaction est assez limitée surtout pour les populations plus fragiles.

Le discours le plus visible, comme partout au Maroc en général, insiste surtout sur la touristification du patrimoine et pas suffisamment sur la création de politiques pédagogiques, d'intégration et de valorisation de ce patrimoine. Le patrimoine est entendu souvent comme une espèce de marchandise, de valeur artistique et historique, bien entendu, mais avec une valeur pour le développement économique régional.

L'intervenant, non institutionnel, le plus actif au niveau local, à Safi, pendant les années du Protectorat Français a été sans aucune doute le Syndicat d'Initiative et du Tourisme. Cet organisme avait des objectifs annuels établis, qu'il transmettait aux autorités locales et nationales, qui concernaient la demande d'intervention sur les éléments patrimoniaux. Il se prononçait activement sur les politiques adoptées par l'administration institutionnelle, en demandant souvent une réflexion et un changement de stratégie visà-vis de ses intentions de dynamisation économique de la ville à travers la mise en valeur du patrimoine ce qui était vue comme une attraction pour le tourisme :

Il est indispensable, tant pour la valeur de nos monuments historiques que pour la beauté et la vie même de Safi au moment où le développement de son port et de ses ressources, où l'achèvement de la route de corniche vers Mazagan et la construction d'un grand hôtel des maintenant approuvé par les Services compétents de la Résidence, vont amener un afflux de touristes tant de Marrakech que des circuits Nord-Africains, il est indispensable de ne pas saccager une des plus belles choses de Safi et de la côte marocaine et la première porte où se porte l'œil du visiteur ${ }^{13}$.

29 Cette lettre se rapportait à la valorisation du Château de Mer, face au projet de la voie ferrée en discussion. Le Château de Mer était ainsi considéré comme la première porte pour le visiteur en arrivant à Safi, même si à l'époque (1930) il était encore englouti par des maisons et magasins. On revient aussi sur la question de ce patrimoine en relation avec la mer, avec les activités portuaires qui constituaient une grande pression sur les politiques locaux.

Si du point de vue des communautés locales, le patrimoine est encore surtout vu comme une ressource pour son territoire, du point de vue institutionnel les questions idéologiques se manifestent aussi clairement. Dès le temps du Protectorat, le choix des éléments patrimoniaux n'a pas été innocent et montre bien l'intention des colonisateurs de s'emparer des symboles de pouvoir du pays. Les séries de cartes postales, qui ont été produites à cette période, démontrent clairement l'intérêt que la gouvernance française a eu pour le patrimoine architectural, notamment pour le patrimoine d'origine portugaise au Maroc.

31 Les images reproduites en série, en identifiant le patrimoine portugais au Maroc, étaient surtout celles qui pourraient nourrir l'idée d'un empire glorieux. Le discours produit par ces cartes postales est celui d'un domaine monumental européen, face au Maroc pittoresque des indigènes. La représentation pictographique exprime et articule les idéologies impérialistes, qui confèrent une espèce de continuité entre le passé colonial et la colonisation moderne. 


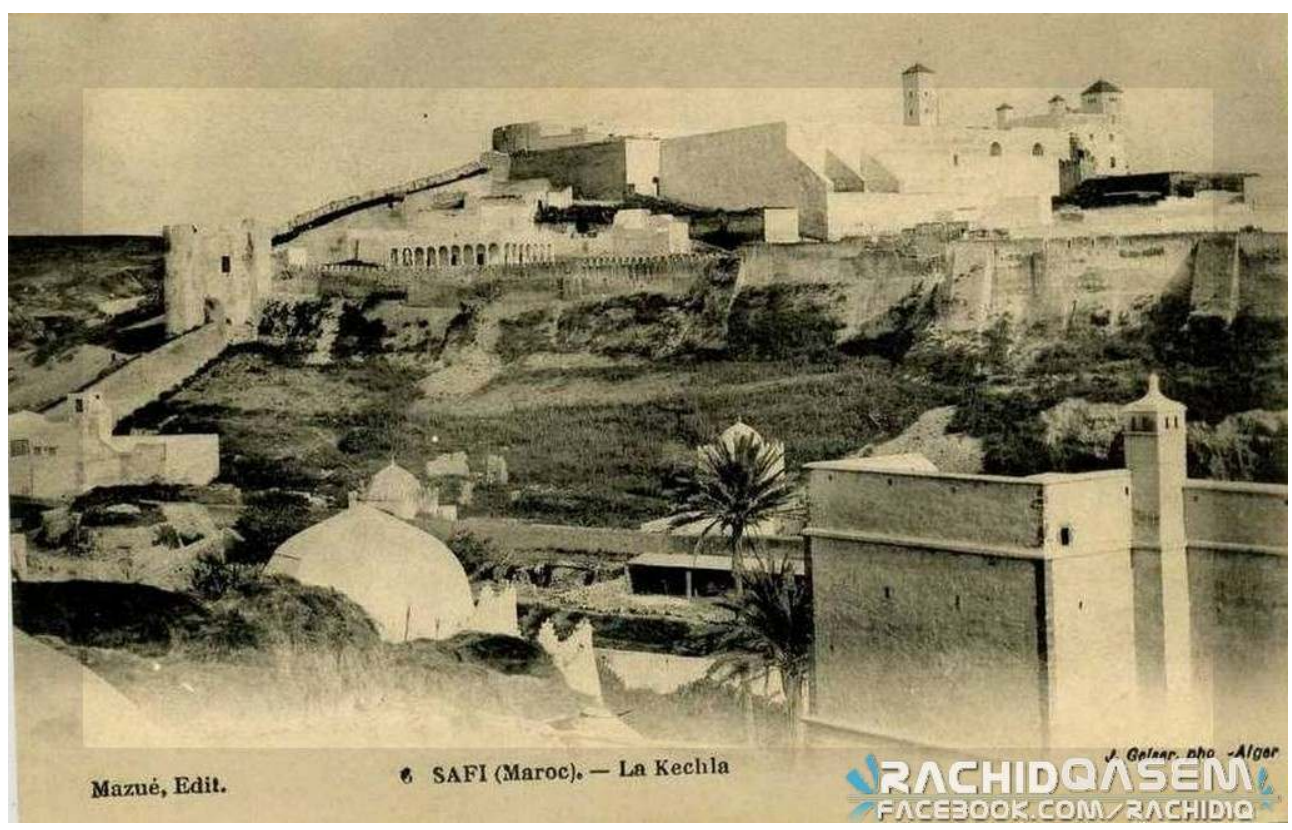

Pour le Maroc colonial, la valorisation de ce patrimoine se faisait surtout par la distinction et non par le contact culturel. Henri Terrace a adopté cette philosophie : « Les architectes portugais n'avaient rien à apprendre de ces forteresses archaïques, mal adaptées au terrain et à peu près incapables de résister au canon $»^{14}$. Le passé portugais correspondait, en fait, à quelque chose de monumental et de caractère strictement européen par opposition à la pittoresque scène indigène traditionnelle.

Ce qu'on reconnaît aujourd'hui comme patrimoine a obtenu ce statut à travers un processus. Chaque classification patrimoniale est donc une production sur laquelle interviennent plusieurs agents, des facteurs sociaux, économiques, culturaux, dans un rapport direct avec les discours dominants qui structurent le pouvoir. Ces discours cherchent plusieurs fois à fabriquer des représentations culturelles qui permettent de guider les communautés pour qu'elles se revoient dans une identité plutôt homogène, dans une identité culturelle de dimension nationale.

Les effets de la colonisation du territoire se sont réfléchis dans la politique culturelle avec le soutien d'un traditionalisme exacerbé. L'administration du Protectorat a essayé d'établir un régime de contrôle qui envisageait d'assurer un équilibre entre colons et indigènes. Un enrichissement culturel de la communauté indigène par la préservation des valeurs traditionnelles était évoqué, ce qui conduirait au développement national, en même temps que les frontières de l'altérité étaient établies.

La conception des valeurs patrimoniales a été ainsi influencée par ces notions de culture et d'identité présentes dans la pensée coloniale. La dichotomie colon/indigène s'est traduite clairement dans l'urbanisme pratiqué par le Protectorat. Dans le dessin des villes, le colon s'est trouvé face à face avec la problématique du patrimoine. Les notions de patrimoine et de sauvegarde étaient encore en maturation, même à la métropole, et le territoire de l'outre-mer était alors une excellente possibilité d'expérimentation. Cependant, le développement des processus de patrimonialisation a rencontré sur le terrain les questions de cette otherness ${ }^{15}$ qui était une réflexion obligatoire dans sa conception. 

acteurs.

\title{
La négociation de l'espace patrimonial et le cas du Château de Mer
}

\begin{abstract}
«M. Meunier précise que le Portugal s'intéresse vivement au sort de ses anciens monuments et qu'un mouvement touristique pourrait être facilement déclenché dans ce pays en raison de l'importance des rentrées de devises par le tourisme, il semble que la consolidation du Château de Mer et la remise en état des monuments de Safi constituent un investissement rentable ${ }^{18}$.»
\end{abstract}

Comme on a pu le voir précédemment, les notions d'urbanisme et de patrimoine ont évolué en parallèle pendant la période coloniale moderne et ont eu des conséquences importantes dans la dynamique sociale, économique et culturelle des villes marocaines. L'ancienne médina a perdu, d'une certaine façon son urbanité au détriment de la conception coloniale de la modernité, transposée à la ville nouvelle, ainsi dénommée comme l'ensemble des quartiers européens, par opposition aux quartiers indigènes. M. Borely, le Chef du Service des Beaux-Arts, écrivait, en 1926, une note de service à propos de la première action de "Conservation des médina suivant les textes instituant une servitude d'aspect », à Safi, qui montre très bien cette conception :

« Il s'agit d'obtenir des propriétaires d'immeubles de médina qu'ils ne construisent que suivant la tradition, avec des appareillages en pierre du pays (on a beaucoup abusé d'affreux mortiers pour les encadrements de portes à Safi ces derniers années) et suivant les proportions et formes anciennes des sobres décorations, 
etc....

Donc s'opposer à tout aménagement de caractère européen dans les vieilles villes.

$\mathrm{Au}$ contraire, dans les villes neuves, où nous intervenons officiellement ou officieusement, il y a intérêt, pour s'assurer une valeur de contraste et pour d'autres raisons, à faire du nouveau; dans le sentiment d'une architecture dictée par le climat ${ }^{19}$.»

41 façons dans l'approche patrimoniale et dans la gestion urbaine et architecturale. L'ancienne médina s'est transformée souvent dans un espace limité et restreint, pétrifié par les idées de l'authenticité, la tradition, le pittoresque, anachronique à la ville nouvelle avec de nombreuses possibilités d'expansion et d'expression architecturale et urbaine. Cette approche a conditionné, d'une certaine façon, le développement socio-économique des zones historiques et est à la base d'une vision dégradante de ces tissus historiques. Encore aujourd'hui, ce sont les secteurs dits modernes des villes marocaines qui représentent la dynamique socio-économique et l'urbanité (les banques, les tribunaux, les grandes écoles, les bibliothèques, les principales administrations).

Dans les discours des gens de Safi, même de ceux liés à la valorisation de la médina et du patrimoine culturel, les zones des anciens quartiers historiques sont souvent vues comme des zones aux conditions sociales sensibles, qu'il est plutôt déconseillé de fréquenter, peuplées la nuit par les gens de vices néfastes, à l'exemple d'hôtels dits insalubres et de services de restauration considérés de faible qualité, etc.

Les élites qui autrefois habitaient la médina de Safi, l'ont abandonnée en cherchant les avantages de maisons plus adaptées aux besoins de la vie moderne. La médina a perdu son statut et s'est transformée en abri pour des populations moins enracinées avec des maisons délabrées, dans des conditions insalubres. Une grande partie des commerçants de la médina n'habite plus dans cet espace. Ainsi, ce bouleversement dans l'utilisation des zones historiques et dans les pratiques spatiales des communautés montre une tendance à l'abandon et à la sous-valorisation du patrimoine local.

Le dessin de l'espace et les choix urbanistiques créent une série de possibilités de pratiques spatiales, mais aussi des interdictions à celui qui parcourt l'espace et le redéfinit. L'individu transforme les possibilités en des réalités et il transgresse aussi les interdictions, en créant des nouvelles possibilités, en transformant ou en abandonnant quelques éléments spatiaux. L'individu qui parcourt l'espace peut ainsi transformer chaque signifiant spatial en quelque chose d'autre ${ }^{20}$.

Mais si l'acte de marcher crée l'espace, l'acte de dessiner l'espace conditionne ce discours et introduit des discours complémentaires à cette narration. La théorie de la ville représentative de J. Jacobs (1993) considère qu'on peut trouver des messages codés dans l'environnement qui peuvent être lus comme des textes. Dans cette approche on renforce encore l'idée que l'environnement bâti devient un outil discursif. La délimitation du terrain d'étude est ainsi très importante pour comprendre la dynamique spatiale et urbaine, codée dans les processus et politiques de patrimonialisation. Le terrain élu, n'est pas strictement un site, mais un champ de relations sociales signifiantes et de pratiques spatiales déterminantes pour la conception de représentations plurielles et polyphoniques des éléments spatiales et plus spécifiquement du patrimoine. Le terrain urbain de la ville est un terrain particulièrement intéressant parce que la ville est le point d'articulation des relations complexes entre le local et le national, l'informel et l'institutionnel, l'individuel et le collectif. 

Château de Mer, le deuxième élément architectural, objet du classement patrimonial à Safi, en 1922. Le Château de Mer, Ksar el Bahar en arabe, appelé aussi la Maison de la Mer pendant les premières années du Protectorat, ou parfois tout simplement le Château Portugais, était à l'époque de l'installation de l'administration coloniale française à Safi un espace approprié et adapté par les populations locales à leurs besoins, exposé à la croissance spontanée de la ville. Le cas du Château de Mer n'a pas été unique, tous les autres monuments d'origine portugaise à Safi ont été sujets à des processus similaires d'appropriation, avec différentes typologies d'occupation selon les localisations. La Kechla a été occupée par le palais Dar el Sultan, les ruines de la cathédrale ont été converties en hammam, les vestiges de l'église de l'Impasse de Sidi Abd el Krim ont été convertis en maisons, aux remparts de la médina ont été adossés des magasins anglais appelés autrefois le «Cercle International ». Ainsi le Château de Mer, comme on peut le voir dans les cartes postales de l'époque ou dans les documents trouvés dans des archives ${ }^{21}$, a été englouti par des maisons et des commerces.

Les démarches pour le classement, la sauvegarde de ce monument et le dessin urbain de la période moderne réfléchissent la politique culturelle et le discours du pouvoir du Protectorat Français, et ont conditionné fortement les pratiques spatiales des communautés par rapport à cet élément architectural et à sa relation avec la ville et le centre historique.

L'immeuble du Château de Mer, à l'époque ou a été émise une proposition pour son classement comme monument historique, était occupé en son intérieur même et avait plusieurs constructions adossées à ses murs comme je l'ai mentionné ci-dessus. Néanmoins, ces constructions appartenaient à plusieurs propriétaires, très différents :

"L'immeuble appartient à l'État, mais autrefois des particuliers en auraient usurpé diverses parties, cédées ensuite par actes d'adoul ${ }^{22}$, à certaines personnes qui font aujourd'hui valoir leurs droits. Leurs prétentions sont sans importance pour le classement, puisqu'en ce qui concerne les monuments historiques proprement dits, ce qui est le cas en l'espace, le dahir du 13 février 1914, n'a pas prévu qu'il pourrait être allouée une indemnité au profit des propriétaires de ces immeubles.

Ces propriétaires seraient M. de Silva, M. Thami Ould El Hadj Mohamed el Mostari, la Maison anglaise Murdoch, Butler et Cie. Les Habous posséderaient aussi dans le château une chambre au rez-de-chaussée occupée par le tombeau du saint Sidi Tahar ben el $\mathrm{Kebir}^{23}$."

L'administration coloniale a procédé au classement du monument, bien que l'immeuble était encore otage de plusieurs constructions qui interféraient avec cet espace. Les interférences provenaient non seulement de citoyens locaux, mais aussi d'étrangers et d'un espace religieux. Cet espace sacré, le Marabout de Sidi Tahar el Kebir, est la seule construction qui a été préservée jusqu'à nos jours à l'intérieur du Château de Mer.

En 1922, le Maréchal Lyautey a promulgué le dahir portant au classement comme monument historique, le Château de Mer portugais à Safi. Ainsi, l'intérêt pour la protection de cet élément architectural a été légitimé par l'administration coloniale, mais une importante condition au classement de ce monument a été émise :

«Article II - Est d'ores et déjà autorisé dans les conditions prévues par NOTRE DIRECTEUR GENERAL des travaux publics et NOTRE DIRECTEUR GENERAL, de l'Instruction Publique, des Beaux-Arts et Antiquités, le percement d'un tunnel au travers de ce monument pour le passage de voie ferrée du port ${ }^{24}$. » 


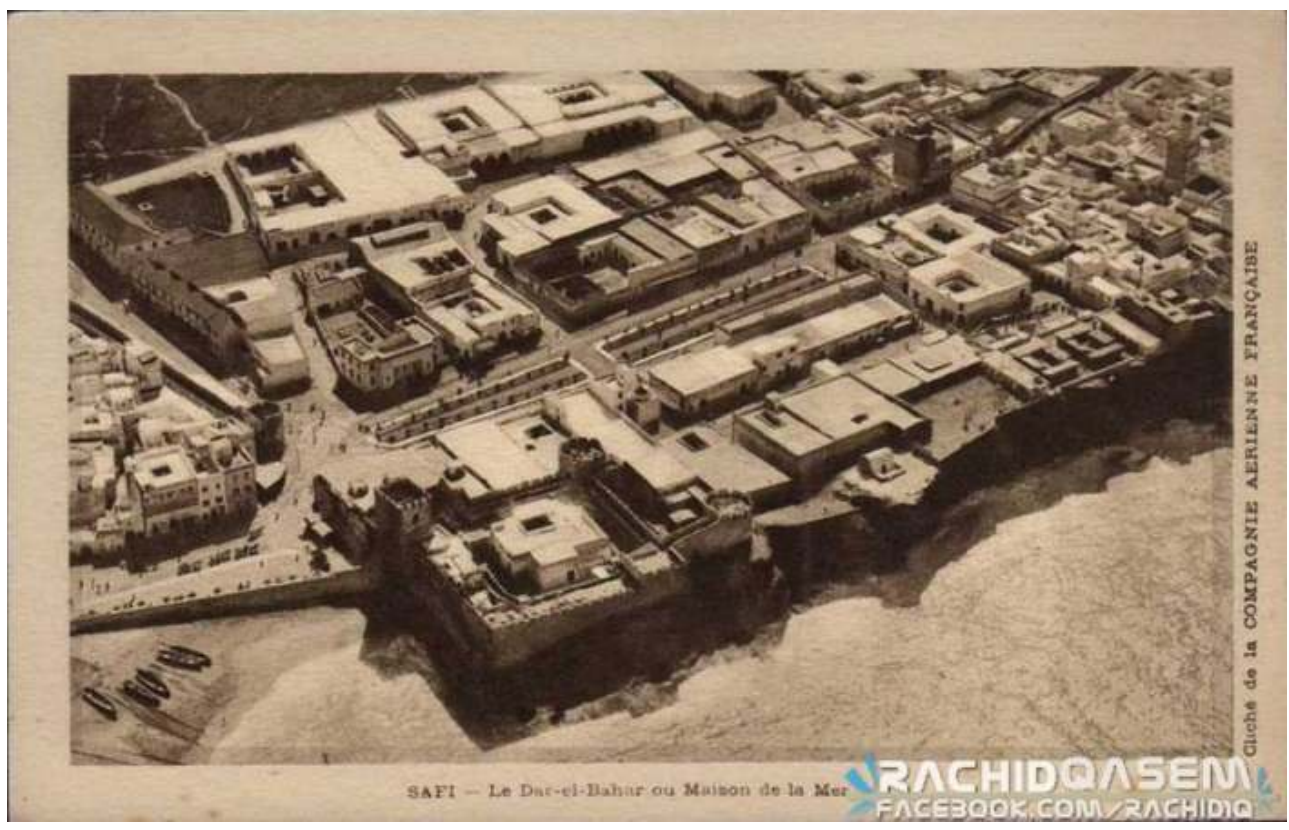

51 L'espace urbain de Safi absorbait donc plusieurs transformations que l'administration coloniale essayait sur le terrain, en altérant profondément son caractère. La valeur du patrimoine ne pouvait pas être promue seule, en oubliant l'aménagement de l'ensemble du territoire et son exploitation face aux intérêts du Protectorat.

52 La question de la ligne ferrée, ainsi que le futur de ce monument, a été le sujet de plusieurs correspondances au long des années pour coordonner les intérêts du Service des Beaux-Arts avec ceux des Services de l'Instruction Publique et des Travaux Publiques, et il a été suivi par d'autres acteurs institutionnels, ou non institutionnels comme le Syndicat de l'Initiative pour le Tourisme ou la Section Historique du Maroc.

Dans le rapport du Lieutenant-Colonel Henry de Castries de sa Mission de 1926 au service de la Section Historique du Maroc, celui-ci identifie les monuments de Safi comme « des vestiges d'architecture plus intéressants que ceux de Mazagan $»^{25}$. En se référant au Château de la Mer (Bordj el-Bahar), il reconnaît un vrai intérêt à cette construction du point de vue architectural et historique, la considérant comme représentative du début de l'occupation portugaise au Maroc. Castries note le besoin de mener une action de réaménagement de l'immeuble :

«J'ai visité Borj el-Bahar et j'ai été frappé de son délabrement et de son état d'abandon : il est envahi par des ménages arabes y logeant par tolérance, et par des commerçants européens ou indigènes qui y ont adossé leurs boutiques; il faudrait se débarrasser de tous ces occupants, soit en les faisant déguerpir, soit en les expropriant, le bordj ainsi dégagé aurait son véritable caractère ${ }^{26}$. »

Ces premiers temps de la patrimonialisation à Safi ont provoqué des discussions sur les usages qui pourraient être admis dans les monuments historiques. La question du tourisme a toujours été centrale. Les politiques patrimoniales se dessinaient sur le terrain d'expérimentation que constitue la colonie :

"J'ai l'honneur de vous soumettre ma manière de voir au sujet de la construction d'un hôtel de touristes auprès du château portugais à Safi.

Le projet me paraît excellent. Le site est des plus pittoresques, mais il importe de ne 
point l'amoindrir par une construction d'un goût contestable. À qui demandera-t-

on le projet ? Le mettra-t-on au concours ? ${ }^{27}$ " de Safi, le dessin de son trajet a été très discuté, à cause d'une interférence ou proximité qui pourrait, d'un côté, menacer la valeur artistique du monument, mais aussi parce qu'il pourrait, de l'autre, permettre de dégager plus facilement le château de constructions indésirables. Ce qui pourrait, dans une première approche, sembler incompatible avec la protection du monument, a été entendu par certains comme un atout pour sa conservation : "Le tracé adopté - et qu'il n'est pas possible de modifier - laisse intact le château de mer, et a même l'avantage d'assurer son exécution en faisant tomber toutes les constructions privées qui l'entourent actuellement $»^{30}$.

Si d'un côté, la voie ferrée était considérée comme un facteur important de développement de la ville dont la construction n'a jamais été mise en cause dans les documents qu'on trouve dans les archives, il faut dire aussi que quelques voix n'étaient pas favorables à son passage si proche d'un monument historique tel que celui du Château de Mer. Cet itinéraire introduisait une radicale séparation physique entre le monument et la ville. Des questions pertinentes sur l'accès au château ont été posées :

«Je me permets donc de vous demander de faire examiner si, sans rien modifier au tracé adopté, il ne serait pas possible de faire abaisser les rampes (montante et descendante - s'il y en a deux) assez profondément pour qu'on puisse couvrir la tranchée d'une dalle en ciment qu'on n'aurait qu'à recouvrir de terre en adoptant, 
sur cette dalle, là, où, sans doute, on doit faire un ou plusieurs ponts ou passerelles pour la circulation, un passage assez solide pour cet usage ${ }^{31}$.»

Pusieurs dessins ont été étudiés, mais ce qui a été finalement construit et qui reste actif jusqu'à nos jours, est une voie ferrée en surface qui passe à peu de mètres du monument. Le Château de Mer a été progressivement dégagé des immeubles parasites, aux yeux des agents $\mathrm{du}$ Protectorat, mais l'accès au monument est devenu chaque fois plus conditionné, surtout après la clôture introduite dans les années 1950, ce qui a obligé la construction d'un accès piéton souterrain.

61 En 1934, le premier avis de menace d'écroulement du château par la force de la mer a été émis par le Médecin-Directeur du Bureau d'Hygiène :

«L'infirmier du Bureau d'Hygiène a constaté, il y a quelques jours, qu'un énorme bloc de rocher s'était détaché de la falaise qui supporte la terrasse des canons au château de Mer et qu'ainsi l'angle Sud-Ouest du bastion, qui a été mis à la disposition des «Éclaireurs de France », porte à faux au-dessus de la Mer, n'étant plus soutenu que par l'entrecroisement des grosses pierres de taille qui constituent cet angle ${ }^{32}$.»

62 Les démarches de consolidation de la falaise n'ont pas été faites et les fondations dégradées de la façade Sud-Ouest du monument ont cédé face aux puissants courants de la mer. Cet événement a été attribué à l'ouvrage du nouveau port qui aurait changé les courants maritimes, en accélérant la dégradation de la falaise. Une fois que le concessionnaire des travaux du port a été l'office Chérifien des Phosphates (O.C.P.), l'administration du Protectorat a essayé de lui attribuer la responsabilité des travaux de restauration. Malgré l'urgence et la conscience du danger de nouveaux écroulements, les travaux n'ont pas eu de suite à cause de cette responsabilisation de l'O.C.P. qui n'a jamais assumé ce qui lui était demandé par l'administration.

63 Le 28 janvier 1937, une tempête s'est chargée de détruire une partie considérable de la façade Sud-Ouest du monument. À cause de ce nouvel événement, le Directeur Général de l'Instruction Publique, des Beaux-Arts et des Antiquités au Maroc, aussi responsable de l'Inspection des Monuments Historiques, avertit le Résident Général de France au Maroc de la gravité de la situation et de l'urgence de prendre des mesures :

«Depuis lors la situation du Château de Mer n'a fait que s'aggraver. Les murailles qui dominent la brèche sont de plus en plus fissurées et le socle rocheux qui supporte les parties encore intactes du Château apparaît de plus en plus fendillé et ébranlé.

Je me permets d'insister sur l'urgence de la décision à prendre ${ }^{33}$.» 
Figure 3 : Origine inconnue, image postérieure à l'écroulement de 1937, diffusée en ligne https:// www.facebook.com/rachid1q, consulté le 30/03/2013.

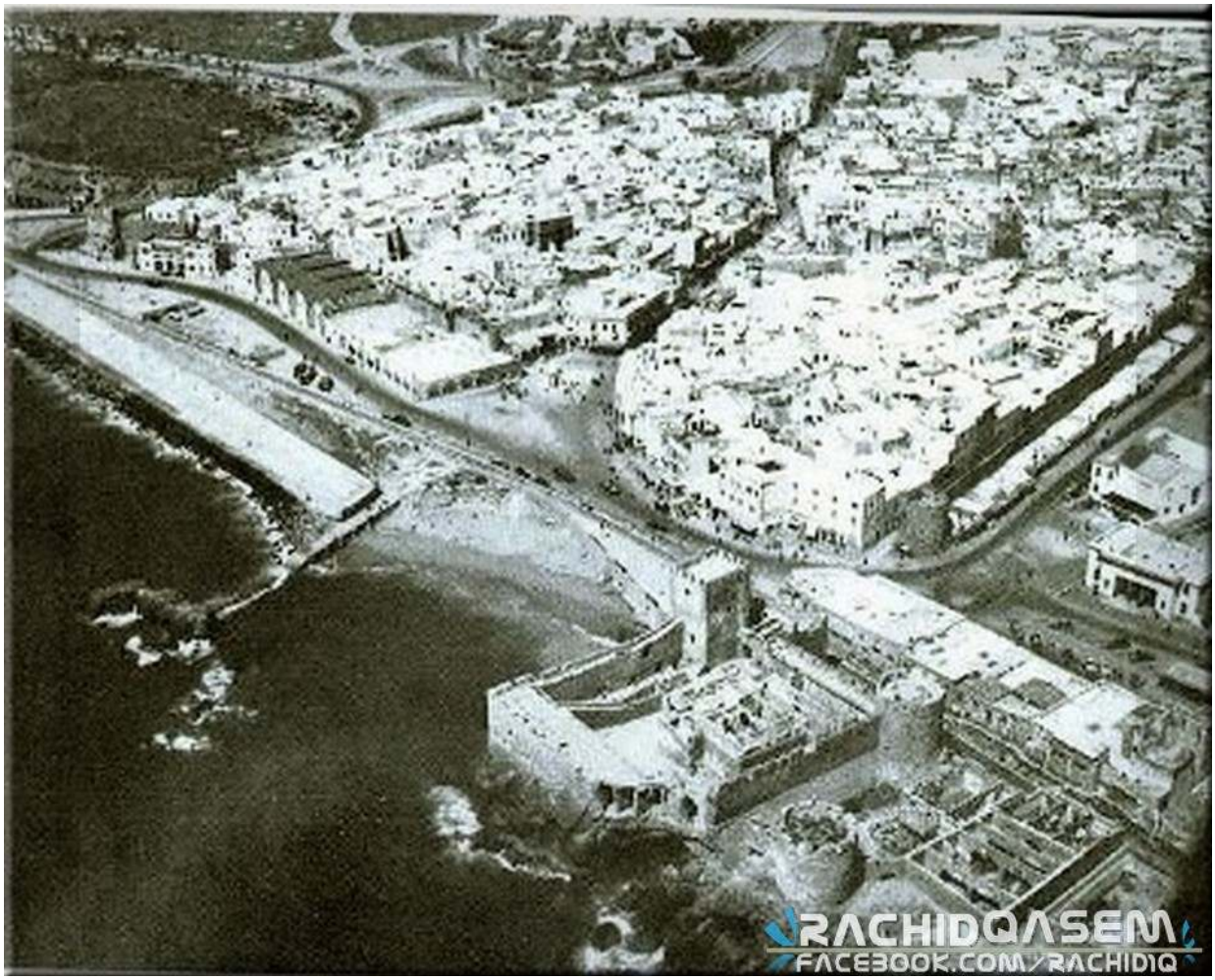

64 Les acteurs locaux étaient très conscients de la gravité de la situation et ont essayé de passer l'alarme à tous les niveaux de l'administration coloniale, auprès du Ministre Plénipotentiaire notamment, en utilisant plusieurs arguments, comme par exemple, celui de la restauration urgente d'un élément de référence non seulement pour la ville de Safi, mais également pour tout le Maroc:

« Si cet ouvrage n'est pas exécuté - non pas seulement en face de la brèche actuelle mais sur tout le fond du château - toute la forteresse est destinée à disparaître, morceau par morceau. En d'autres parties de l'enceinte la roche qui supporte la courtine est déjà en surplomb et on entend la mer s'engouffrer dans les galeries creusées sous le château lui-même. Chaque grande tempête abattra par pan entiers la plus belle forteresse du Maroc ${ }^{34}$. »

Malgré tous les avertissements, rien n'a été fait tout au long des années suivantes pour garantir la sécurité du lieu, la consolidation du front de mer ou la restauration de cet important monument. La Deuxième Guerre mondiale a encore retardé toute intervention possible. Tous les travaux ont été suspendus pendant cette période. Néanmoins, plusieurs documents montrent que la question du Château de Mer n'a pas été oubliée. Si le château était vu comme un important monument dans le contexte de la préservation du patrimoine du Maroc, il n'était pas moins important du point de vue des relations diplomatiques avec le Portugal :

«Dans nos relations avec le Portugal, nous ne manquons jamais de rappeler le soin que les autorités du Protectorat, fidèles à la tradition instaurée par le Maréchal Lyautey, apportent à la conservation des vestiges portugais du Maroc. La disparition même partielle du château de mer de Safi nous ferait perdre la face devant l'opinion et le Gouvernement portugais ${ }^{35}$. » 
correspondances échangées au long de toutes ces années ont eu un agrément généra pour la restauration de ce patrimoine d'intérêt local, national et supra-national. Cependant, le budget pour ces travaux, ainsi que pour le dégagement de quelques constructions qui envahissaient encore le château et ses zones de servitude n'a jamais été attribué par aucun service administratif du Protectorat. En 1948, M. Thabault, le Directeur de l'Instruction Publique rapportait au Contrôleur Civil, Chef du Territoire de $\mathrm{Safi}^{36}$, la constatation de l'état critique du monument faite par les figures portugaises, David Lopes et Reinaldo dos Santos, ainsi que leur proposition d'intervenir auprès du gouvernement portugais, ce qui a été refusé par le Protectorat. Jusqu'en 1952, les promesses faites au gouvernement portugais sont encore évoquées sans que rien ne soit exécuté. Si ces promesses avaient pour intérêt de nourrir une relation diplomatique cordiale avec le Portugal, il était aussi question de ne pas discréditer la France en tant que puissance politique dans le territoire colonial :

«Outre l'intérêt réellement historique que présente ce monument, il convient d'éviter que des offres de participation financière du Gouvernement Portugais à la restauration d'un des plus précieux souvenirs de son action passée au Maroc ne soient encore présentées, ce qui ne manquerait pas de nuire à notre prestige de puissance protectrice $[. . .]^{37}$,

archives ne sont pas tout simplement des sources d'informations, mais elles doiven être comprises de façon critique, parce qu'elles ne correspondent pas à une pensée uniformisée, mais à une fraction de discours sélectionnés. Beaucoup de voix sont absentes des archives, surtout les voix de contestation. Malgré cette vision d'une uniformité du discours sur l'option consensuelle de conservation et restauration du Château de Mer, on trouve des résistances à ce discours dans des propositions dissonantes de propriétaires de constructions faisant parti de l'ensemble du monument.

Le Protectorat a assumé une politique d'épuration de toute interférence sur le monument, même si les populations avaient une vision différente sur l'occupation de cet espace. Un document intéressant de contestation est resté, en tout cas, dans les archives marocaines. C'est le cas d'un petit article d'opinion intitulé "Le château (?) de mer fait beaucoup parler de lui» publié le 15 avril 1952 dans le Petit Marocain et qui s'opposait grandement aux travaux de restauration à effectuer :

«[...] Comment va-t-on dépenser des dizaines de millions pour rafistoler ces vieilles pierres qui ne tiennent plus, alors que des centaines de braves gens sont sans logis ou tellement mal logés qu'il vaux mieux ne pas en parler? C'est pour ces ruines que l'on va mettre dans la rue d'honnêtes commerçants qui donnent satisfaction à leurs clients depuis des années? [...]

Alors, mais alors seulement, on pourra peut-être penser à démolir certaines boutiques et le Château (?) de Mer, par la même occasion, pour créer une plage à Safi qui n'en a plus. Place du R'bat-Plage sera certainement plus appréciée que Place du R'Bat-Château de Mer.

Voilà ce que disent les Safiots, et franchement, nous les approuvons complètement ${ }^{38}$ ."

Selon l'article cité, les safiots n'étaient pas d'accord avec les options de l'administration coloniale. Il serait incorrect d'assumer que l'opinion de tous les safiots était celle exprimée dans cet article, néanmoins il nous est très utile pour comprendre que les processus et politiques de patrimonialisation n'ont pas été des actions acceptées et désirées de manière consensuelle. Malgré l'opposition déclarée publiquement, la réponse d'Henri Terrace à cette question a dévalorisé complètement l'article et finalement, l'année suivante, les travaux ont commencé au Château de Mer. 
70 Les travaux de restauration ont dépassé l'année de l'indépendance du Maroc. En 1956, une importante réunion interministérielle a été tenue pour discuter le besoin de se rejoindre dans l'effort de récupération du monument, vu la dimension et la difficulté des travaux. L'état de dégradation de la falaise imposait une intervention de dimension beaucoup plus importante que celle prévue auparavant. Les représentants qui ont participé à cette réunion intégraient des marocains, aussi bien que des français : par le Ministère de l'Instruction Publique M. Abdelkader Kahhak, M. Nekrouf et M. Meunier ; par le Ministère de l'Intérieur M. Amar Benouzekri ; par le Ministère de l'Urbanisme M. Cousin; par le Ministère des Travaux Publics M. Dupont et M. Bauduret; et par les Chemins de Fer du Maroc M. d'Inguimbert. La transition des postes des responsables pour l'urbanisme et le patrimoine n'a pas été immédiate au moment de l'indépendance. Les actions ont continué sans changements profonds les premières années et les documents qui figurent dans les archives, même après 1956, continuent à être entièrement en langue française.

71 Les travaux ont avancé jusqu'à 1960, quand les crédits pour la restauration ont été épuisés en empêchant sa suite. En décembre 1961, M. Sefrioui, le Chef du Service des Monuments Historiques, des Arts et du Folklore informait M. le Gouverneur de la Ville de Marrakech de la fin des travaux au Château de Mer :

«[...] j'ai le plaisir de vous annoncer que les dits travaux touchent à leur fin. La ville de Safi est d'ores et déjà dotée d'un superbe monument $\mathrm{du} \mathrm{XV}^{\mathrm{e}}$ siècle qui enchantera les touristes. Pour que ce monument soit réellement en valeur, il serait souhaitable que la décision de la démolition des boutiques qui cachent la façade du château devienne exécutoire. La Municipalité qui avait commencé à faire le nécessaire pour leur évacuation semble se désintéresser de ce problème. Il s'agit d'une mesure d'embellissement et de mise en valeur esthétique de Safi de première importance.

Je vous serais infiniment reconnaissant si vous vouliez bien donner les ordres nécessaires afin que les travaux de démolition des boutiques soient entrepris dans les meilleurs délais. Nous aurions aimé que le Château de Mer soit inauguré par Sa Majesté lors de son prochain séjour à Safi ${ }^{39}$. »

\section{Conclusions}

« La métaphore du centre vide identifiée par Françoise Choay en ce qu'elle propose de considérer « la ville comme un système non verbal d'éléments signifiants qui se révèle à l'état pur dans les sociétés closes à l'évolution lente" s'applique bien évidemment, aux fragments de villes comme les médinas. Car celles-ci dans leur mort lente ou par leur métamorphose, ne cessent d'interroger sur ce qui adviendra des cultures et des mémoires et sur l'inéluctabilité des bifurcations historiques ${ }^{40}$. »

72 Le classement du Château de Mer n'a pas posé de problèmes du point de vue de la reconnaissance de cet élément architectural en tant qu'élément exceptionnel de grande valeur historique et artistique. Au-delà de cette reconnaissance, le test sur le terrain de certaines pratiques et politiques patrimoniales, de protection et mise en valeur et son rapport avec l'urbanisme et les pratiques spatiales ont conditionné l'évolution architecturale et urbaine du lieu, à travers des plans d'aménagement.

Le drapeau marocain, bien visible sur le bastion Nord-Est du château à partir de l'Avenue Hassan II, de la Place du R'bat, ou bien de la place de l'ancienne médina, démontre bien l'importance de la valeur symbolique de cet immeuble, même s'il n'est plus ouvert au publique. 
74 Les pouvoirs successifs se sont appropriés ce symbole de souveraineté, mais les difficultés pour la mise en place d'une politique de sauvegarde efficiente semblent encombrer le monument depuis une centaine d'années, depuis les premières démarches pour son classement.

Les pratiques spatiales quotidiennes tournent le dos à ce monument, comme conséquence des choix politiques et des métamorphoses imposées au lieu. L'imposition d'une épuration de toutes autre pratiques et usages a isolé le Château de Mer dans une espèce d'immobilité dans le temps monumental. Les mémoires restent matérialisés dans les souvenirs de la ville ou dans les discours, même si quelques safiots n'ont jamais osé rentrer dans ces espaces patrimonialisés.

Même si le Borj Sud-Ouest du Château de Mer tombe à nouveau en ruine, englouti par l'océan, il est cependant l'image la plus représentée par les artisans de la poterie de Safi, qui le nomment le Borj Asfi (le Bastion de Safi). Le Château de Mer reste dans la mémoire collective safiote, même si les pratiques spatiales ont beaucoup changé pendant les dernières décennies. La mémoire de ce monument va au-delà de sa valeur historique et artistique, c'est le symbole de la forte relation de Safi avec sa mer qui nourrit l'imaginaire collectif des communautés. Si le Château de Mer est la carte de visite de Safi, évoquée par l'administration coloniale, il est aussi le symbole de l'orientation de la ville vers la mer.

\section{BIBLIOGRAPHIE}

BAKHTINE, Mikhail, La Poétique de Dostoïevski. Paris : Seuil, 1970.

BHABHA, Homi K., The location of culture. London : Routledge, 1994.

CERTEAU, Michel de, The practice of everyday life. Berkley : University of California Press, 1984.

CHOAY, Françoise, L'allégorie du patrimoine Paris : Editions du Seuil, 1988.

FADILIL-TOUTAIN, Rima, Historique de la sauvegarde par la législation in Cattedra Raffaele et al., Patrimoines en Situation. Constructions et Usages en différents contextes urbains. Rabat : Centre Jacques Berque, 2010.

GAVARI-BARBAS, Maria et Guichard-Anguis, Sylvie, Regards croisés sur le patrimoine dans le monde à l'aube du XXIe siècle, Paris, PUP, 2003.

HERZFELD, Michael, A Place in History : Social and Monumental Time in a Cretan Town. New Jersey : Princeton University Press, 1991.

HOBSBAWM, Eric, The Invention of Tradition. Cambridge : Cambridge University Press, 2000

KING, Anthony D, Colonial urban development : culture, social power, and environment. London : Routledge, .2007.

LIMA, António P. ; Sarró, Ramon (org.) (2006) ; Terrenos Metropolitanos : Ensaios sobre produção etnográfica. Lisboa : Imprensa de Ciências Sociais.

LOW, Setha M. (ed.) ; Theorizing the City : The New Urban Anthropology Reader. New Jersey : Rutgers, 1999. 
JACOBS, J. ; « The city unbound : Qualitative approaches to the city. » Urban Studies 30, 1993, pp. 827-848.

KING, Anthony D., Colonial urban development : culture, social power, and environment. London : Routledge, 2007.

TROIN, Jean-François ; Maroc : Régions, Pays, Territoires. Paris : Maisonneuve et Larose, 2002.

WRIGHT, Gwendolyn ; Tradition in the Service of Modernity in French Colonial Cities, 1900-1930 in Tensions of empire : colonial cultures in a bourgeois world. Berkley : University of California Press, 1997.

\section{NOTES}

1. GIRARD, Allocution de réception de la visite de M. Lacoste, Ministre Plénipotentiaire, Délégué à la Résidence Générale, à Safi. Nantes, Centre des Archives Diplomatiques de Nantes, 27 Septembre 1948.

2. Auteur inconnu, Rapport sur la Réorganisation du Territoire de Safi, Nantes, Centre des Archives Diplomatiques de Nantes, 27 Septembre 1948. p. 1.

3. Mahakma désigne le tribunal.

4. Auteur inconnu, Fiche sur l'organisation du Contrôle Urbain à SAFI (Affaires Marocaines Arrondissements), Nantes, Centre des Archives Diplomatiques de Nantes, 30 Mai 1955. p. 1.

5. TROIN Jean-François, Maroc : Régions... op. cit., p. 178.

6. TROIN Jean-François, Maroc: Régions, Pays, Territoires. Paris, Maisonneuve et Larose, 2002. p. 173.

7. TROIN Jean-François, Maroc : Régions... op. cit., p. 176.

8. J'utilise le concept de polyphonie de Michel Bakhtin.

9. CERTEAU Michel de, The practice of everyday life, Berkley, University of California Press, 1984. p. 92.

10. GAVARI-BARBAS Maria et GUICHARD-ANGUIS Sylvie, Regards croisés sur le patrimoine dans le monde à l'aube du XXIe siècle, Paris, PUP, 2003, p. 29.

11. AAVV., Safi et sa région. Safi, Chambre Mixte Française d'Agriculture, de Commerce et d'Industrie de Safi, Nantes, Centre des Archives Diplomatiques de Nantes, 1952. p. 11-13

12. Auteur inconnu, lettre de L'Inspecteur Régional à Monsieur le Chef du Service des Beaux-arts et Monuments Historiques, Rabat, Direction du Patrimoine Culturel, 5 Novembre 1921, p. 1-3.

13. LEBERT, lettre du Président du Syndicat d'Initiatives et de Tourisme Régional de Safi à Monsieur le Chef du Service des Beaux-arts, Rabat, Direction du Patrimoine Culturel, 15 Janvier 1930, p. 2-3.

14. TERRACE Henry, "Note sur les contacts artistiques entre le Maroc et le Portugal du Xve au XVIIe siècle" in Robert Ricard, Mélanges d'études luso-marocaines dédiés à la mémoire de David Lopes et Pierre de Cénival, 1945, p. 404.

15. J'utilise le concept de otherness dans le sens de la production de l'altérité, tel qu'il est utilisé par Hommi Bhabha

16. CERTEAU Michel de, The practice of everyday life, Berkley, University of California Press, 1984. p. 94.

17. CERTEAU Michel de, The practice of everyday life, Berkley, University of California Press, 1984. p. 95.

18. SMAILI Abderrahman Alaoui, Procès-verbal de la réunion du 14 Août 1956 pour les travaux de conservation du Château de Mer de Safi, Rabat, Direction du Patrimoine Culturel, 28 Décembre 1956, p. 2. 
19. BORELY, Note pour M. Teillet - Rolle de notre service - $1^{\circ}$ Conservation des médina suivant les textes instituant une servitude d'aspect, Rabat, Direction du Patrimoine Culturel, 15 Janvier 1930, p. 2-3.

20. CERTEAU Michel de, The practice of everyday life, Berkley, University of California Press, 1984. p. 98.

21. Les archives consultés sont l'Archive de la Direction du Patrimoine Culturel du Ministère de la Culture à Rabat et le Centre des Archives Diplomatiques de Nantes.

22. L'adel (pluriel en arabe : adoul) est le notaire de droit musulman. Au Maroc, il a la charge des affaires relatives au droit personnel.

23. PAUTY, lettre du Chef du Service de Beaux-Arts et Monuments Historiques à Monsieur le Secrétaire Général du Protectorat, s/c de M. le Directeur Général de l'Instruction Publique, des Beaux-Arts et Antiquités, Rabat, Direction du Patrimoine Culturel, 18 Octobre 1922.

24. LYAUTEY, Dahir du 7 Novembre 1922 (17 Rebia I 1341) portant classement comme monument historique du Château de mer portugais à Safi, Rabat, Direction du Patrimoine Culturel, 7 Novembre 1922 .

25. CASTRIES Henry, Rapport de la Mission de 1926. Nantes, Centre des Archives Diplomatiques de Nantes, 15 Février 1927. p. 11.

26. CASTRIES Henry, Rapport de..., op.cit., p. 12.

27. BORÉLY, lettre au Chef des Services Municipaux de Safi, s/c de M. le Contrôleur Civil, chef de la circonscription des Abda Ahmar, s/c de M. le Secrétaire Général du Protectorat (Contrôle des Municipalités), s/c M. le Directeur Général de l'Instruction Publique, des Beaux-Arts et des Antiquités, Rabat, Direction du Patrimoine Culturel, 10 Décembre 1925.

28. Règlement d'Aménagement des Abords du Château de Mer Portugais, Rabat, Direction du Patrimoine Culturel, Novembre 1927.

29. Auteur inconnu, programme des travaux du Syndicat d'Initiative et de Tourisme de Safi pour 1928, présenté au Conseil Supérieur du Tourisme, Rabat, Direction du Patrimoine Culturel, 1928.

30. JOYANT, lettre de l'Inspecteur Général des Ponts et Chaussés, Directeur Général des Travaux Publics du Maroc à Monsieur le Chef du Service des Beaux-Arts et Monuments Historiques, Rabat, Direction du Patrimoine Culturel, 27 Février 1932, p. 1-2.

31. Auteur inconnu, lettre de Monsieur le Directeur de l'Administration Municipale - Résidence Générale -, à M. le Directeur Général de l'Instruction Publique, des Beaux-Arts et des Antiquités, Rabat, Direction du Patrimoine Culturel (sans date, en réponse au lettre du 11 Mars 1932).

32. BOHTN, lettre du Médecin-Directeur du Bureau Municipal d'Hygiène à M. le Chef des Services Municipaux de la Ville de Safi, Rabat, Direction du Patrimoine Culturel, 5 Avril 1934.

33. RIBAUT, lettre de Monsieur le Directeur Général de l'Instruction Publique, des Beaux-Arts et des Antiquités à M. le Résident Général de France au Maroc, Rabat, Direction du Patrimoine Culturel, 1937.

34. Auteur inconnu, rapport d'une inspection au Château de la Mer pour le Ministre Plénipotentiaire, Rabat, Direction du Patrimoine Culturel, Novembre 1937.

35. RICARD Robert, lettre du Directeur de l'Inspection Publique au Maroc à M. le Commissaire Résident Général, Rabat, Direction du Patrimoine Culturel, 9 Juin 1941.

36. THABAULT, lettre du Directeur de l'Instruction Publique au Contrôleur Civil, Chef du Territoire de Safi, Rabat, Direction du Patrimoine Culturel, 20 Juillet 1948.

37. HAUTEVILLE, lettre de M. le Général d'Hauteville, Chef de la Région de Marrakech à M. le Directeur de l'Instruction Publique (Service des Monuments Historiques), Rabat, Direction du Patrimoine Culturel, 12 Avril 1952.

38. Auteur inconnu, extrait de l'article «Le château ( ?) de mer fait beaucoup parler de lui », Petit Marocain, transmis à M. le Directeur de l'Institut des Hautes Études Marocaines, Henri Terrasse, Rabat, Direction du Patrimoine Culturel, 15 Avril 1952. 
39. SEFRIOUI, lettre du Chef du Service des Monuments Historiques, des Arts et du Folklore à M. le Gouverneur de la Ville de Marrakech, Rabat, Direction du Patrimoine Culturel, 20 Décembre 1961.

40. BOUMAZA Hadir, «Expérience occidentale et construction maghrébine d'une approche du patrimoine" in GAVARI-BARBAS Maria et GUICHARD-ANGUIS Sylvie, Regards croisés sur le patrimoine dans le monde à l'aube du XXIe siècle, Paris, PUP, 2003, p. 118.

\section{RÉSUMÉS}

Safi, port arabe sous protectorat portugais dès la fin du XVe siècle, a connu une histoire singulière au Maroc. Cet article revient sur la longue histoire (un siècle) de patrimonialisation des principaux monuments de Safi. Il montre la façon dont les pouvoirs successifs se sont emparé de ces édifices et ont sans cesse dus chercher un équilibre entre urbanisme moderne et préservation des édifices anciens. Le cas du château de mer est étudié spécifiquement. (Résumé rédigé par la rédaction).

Safi, Arabic port, Portuguese protectorate since the end of $15^{\text {th }}$ century, had a unique history in Morocco. The paper examine the last century, which saw attempts of patrimonalization of the most important buildings in Safi. It shows how political powers had to balance between modern town-planning and preservation of old buildings. It examines the sea-castle case in particular. (Abstract written by the journal).

\section{INDEX}

Mots-clés : histoire des techniques, Maroc, époque moderne, époque contemporaine, château, patrimoine, urbanisme

Keywords : history of technology, Morocco, early moderns, modern, castle, heritage, townplanning

\section{AUTEUR}

\section{ANA SOFIA NENO LEITE}

Doctorante, CES/Université de Coimbra et FLSH/université Cadi Ayyad de Marrakech 